\title{
Nested soft-collinear subtractions for NNLO calculations
}

Raoul Röntsch*†

Institute for Theoretical Particle Physics, Karlsruhe Institute of Technology

E-mail: raoul.roentsch@kit.edu

\begin{abstract}
I present a subtraction scheme for next-to-next-to-leading order (NNLO) QCD calculations, based on the residue-improved sector decomposition method. The subtraction scheme is characterized by the independent treatment of soft and collinear limits, leading to a straightforward procedure to remove singularities using nested subtractions. The scheme is fully local and results in relatively compact expressions for the singularities, allowing the cancellation of the poles to be checked explicitly. Following this, all matrix elements may be evaluated in four dimensions. I present some of the details of the subtraction scheme applied to color singlet production at NNLO QCD, based on the work of Ref. [1]. I also present results from a proof-of-concept calculation and comparison, showing that the subtraction scheme is capable of delivering numerically stable results for NNLO QCD calculations.
\end{abstract}

13th International Symposium on Radiative Corrections 24-29 September, 2017

St. Gilgen, Austria

\footnotetext{
* Speaker.

${ }^{\dagger}$ Based on work with F. Caola and K. Melnikov [1]
} 


\section{Introduction}

One of the challenges that arises in performing calculations at next-to-next-to-leading order (NNLO) in quantum chromodynamics (QCD) is handling the infrared singularities which appear at intermediate stages of the calculation while maintaining a complete description of the final state. Infrared singularities arise from either virtual corrections or from real emission corrections. The former give rise to poles which are explicitly present in the amplitudes, while the poles in real corrections originate from regions of phase space where partons become unresolved, and therefore are only manifest after integrating over the phase space. It is necessary to devise a procedure to treat the infrared singularities in such a way as to allow fully differential results to be computed.

The past few years have seen impressive progress in the development of such techniques. This work may be broadly divided into two categories. The first is slicing-based methods, such as $q T$ slicing [2,3] or $N$-jettiness slicing [4, 5]. The basic idea in these approaches is to divide the emission phase space into two kinematic regimes. In the first, the real emissions are unresolved. The phase space can be treated as Born-like, while the amplitudes can be calculated in a soft-collinear approximation. In the second, the real emissions are always resolved, and the calculation can be treated as at next-to-leading order (NLO). The second category is subtraction schemes. Here the idea is to identify a subtraction term which reproduces the singular structure of the real emission amplitude. By construction, the difference between the real emission amplitude and the subtraction term has no singularities, and may be integrated in four-dimensional phase space to give a finite result. One then needs to add back the subtraction term integrated over the $d$-dimensional phase space; this term contains the explicit infrared singularities. There are a number of different subtraction schemes, including antenna subtractions [6], sector decomposition-based approaches [7, 8, 9], projection-to-Born [10], and CoLoRFullNNLO subtraction [11].

Both slicing and subtraction methods have proven highly successful, and have allowed the calculation of fully differential NNLO QCD results for a large number of phenomenologically interesting production processes at the Large Hadron Collider (LHC). Nevertheless, there is room for improvement. Current implementations of subtraction schemes are complicated and consequently require substantial runtimes to produce stable results. Moreover, infrared singularities have clear physical origins, which are often obscured in the development of the subtraction scheme. Slicing methods, on the other hand, require the introduction of an unphysical slicing parameter, and considerable effort must be taken to ensure that the final results are independent of this parameter. In trying to improve NNLO subtraction schemes, one may look to the status of next-toleading order (NLO) calculations for guidance. At this order, subtraction schemes exist which are fully local, process-independent, clearly expose the physical origin of singularities while also allowing their explicit analytic cancellation, and allow all amplitudes to be evaluated in four dimensions $[12,13,14]$. Developing a NNLO subtraction scheme with the above properties would greatly improve the stability and speed of NNLO calculations.

In these proceedings, I will summarize some recent progress in this direction [1] within the framework of residue-improved sector decomposition. The central idea of this framework, developed in Ref. [7], is to extend the highly successful FKS subtraction scheme [12, 13] - which is valid for NLO calculations - to NNLO by using sectors to separate overlapping divergences. However, in previous implementations of this method, the expected simplification of terms on recombining 
sectors is not apparent. The approach proposed in Ref. [1] focuses on gauge-invariant matrix elements and makes use of the color coherence property of QCD, allowing the decoupling of the soft and collinear limits. This leads to relatively compact expressions once the sectors are recombined and an explicit (although numerical) cancellation of the poles for different kinematic structures. Moreover, it allows all matrix elements to be evaluated in four dimensions.

The remainder of this proceeding is organized as follows. In Sec. 2, I will outline the subtraction scheme at NLO as a precursor to the NNLO calculation, which is discussed in Sec. 3. I present some results from a proof-of-concept computation in Sec. 4, and conclude in Sec. 5.

\section{Next-to-leading order calculation}

In this section, I will illustrate the subtraction scheme by considering the NLO QCD corrections to color singlet production. At this order, the method is identical to FKS subtraction $[12,13]$. I focus on the real emission corrections, $q_{1} \bar{q}_{2} \rightarrow V+g_{4}$, where $V$ represents the color singlet final state. This contribution can be written as

$$
\mathrm{d} \sigma^{\mathrm{R}}=\frac{1}{2 s} \int\left[\mathrm{d} g_{4}\right] F_{L M}(1,2,4) \equiv\left\langle F_{L M}(1,2,4)\right\rangle,
$$

introducing the averaging sign $\langle\ldots\rangle$ to indicate integration over the final state phase space. In the above equation, $s$ is the partonic center-of-mass energy, the phase space integration measure of the emitted gluon is

$$
\left[d g_{4}\right]=\frac{\mathrm{d}^{d-1} p_{4}}{(2 \pi)^{d-1} 2 E_{4}} \theta\left(E_{\max }-E_{4}\right)
$$

with $E_{\max }$ an arbitrary energy parameter defined in the partonic center-of-mass frame, and

$$
F_{L M}(1,2,4)=\mathrm{dLips}_{V}|\mathscr{M}(1,2,4, V)|^{2} \mathscr{F}_{\text {kin }}(1,2,4, V) .
$$

In the above, $\mathrm{dLips}_{V}$ is the Lorentz-invariant phase space for the colorless particles, including the momentum-conserving delta function; $\mathscr{M}(1,2,4, V)$ is the matrix element for the process $q_{1} \bar{q}_{2} \rightarrow$ $V+g_{4}$, and $\mathscr{F}_{\text {kin }}$ defines an infrared-safe observable.

The real emission contribution $\mathrm{d} \sigma^{\mathrm{R}}$ develops singularities when $g_{4}$ becomes soft and/or collinear to either initial state parton. I introduce soft and collinear operators

$$
S_{i} A=\lim _{E_{i} \rightarrow 0} A, \quad C_{i j} A=\lim _{\rho_{i j} \rightarrow 0} A,
$$

where $\rho_{i j}=1-n_{i} \cdot n_{j}$ and $n_{i}$ is a unit vector that describes the direction of the momentum of the $i$-th particle in $(d-1)$-dimensional space. I then rewrite the real emission corrections as

$$
\begin{aligned}
\left\langle F_{L M}(1,2,4)\right\rangle= & \left\langle S_{4} F_{L M}(1,2,4)\right\rangle+\left\langle\left(C_{41}+C_{42}\right)\left(I-S_{4}\right) F_{L M}(1,2,4)\right\rangle \\
& +\left\langle\left(I-C_{41}-C_{42}\right)\left(I-S_{4}\right) F_{L M}(1,2,4)\right\rangle .
\end{aligned}
$$

The first term in the above equation corresponds to the soft limit, in which the gluon decouples completely and the integration over its phase space can be performed immediately. However, since the soft operator removes the momentum of the gluon from the momentum-conserving delta function inside $F_{L M}(1,2,4)$, an additional parameter is required to provide an upper bound on the gluonic 
energy. This is the reason for introducing the parameter $E_{\max }$. This parameter is arbitrary provided that it is sufficiently large; in practice, we use the partonic center-of-mass energy. The second term of Eq. (2.5) describes the collinear and soft-collinear singularities, in which the gluon decouples either partially or completely. The final term is manifestly finite, all its singularities having been removed through the nested subtraction of the soft and collinear limits, and it may be numerically integrated in four dimensions. The singularities associated with the soft and collinear limits can be made explicit by integrating over the phase space of the emitted gluon in the first two terms. In the special case of color singlet production, the soft and soft-collinear limits cancel and one need only consider the collinear limits. The effect of the collinear operator $C_{41}$ is

$$
C_{41} F_{L M}(1,2,4)=\frac{g_{s, b}^{2}}{E_{4}^{2} \rho_{41}}(1-z) P_{q q}(z)\left(\frac{F_{L M}(z \cdot 1,2)}{z}\right),
$$

where $g_{s, b}$ is the bare QCD coupling, $z=1-E_{4} / E_{1}$, and the splitting function $P_{q q}=P_{q q}^{(0)}+\mathscr{O}(\varepsilon)$. Since the gluonic angles decouple, we can immediately integrate over these to obtain a $1 / \varepsilon$ pole. On the other hand, the function $F_{L M}(z \cdot 1,2)$ still depends on the gluonic energy through $z$, and since $F_{L M}$ is process-dependent we cannot integrate over the energy in general. Nevertheless, it is still possible to demonstrate the cancellation of poles by observing that the collinear singularities must cancel against a redefinition of the pdfs. I therefore rewrite the integral over the gluonic energy as an integral over $z$ and introduce a plus-prescription for the singularity at $z=1$, which makes the singularity explicit. The result is

$$
\begin{aligned}
\left\langle C_{41} F_{L M}(1,2,4)\right\rangle= & -\frac{\left[\alpha_{s}\right] s^{-\varepsilon}}{\varepsilon} \frac{\Gamma^{2}(1-\varepsilon)}{\Gamma(1-2 \varepsilon)} \times\left[-\left(\frac{C_{F}}{\varepsilon}+\frac{3 C_{F}}{2}\right)\left\langle F_{L M}(1,2)\right\rangle\right. \\
& \left.+\int_{0}^{1} \mathrm{~d} z \mathscr{P}_{q q, R}(z)\left\langle\frac{F_{L M}(z \cdot 1,2)}{z}\right\rangle \cdot\right]
\end{aligned}
$$

In the above, $\mathscr{P}_{q q, R}(z)=\hat{P}_{q q}^{(0)}(z)+\varepsilon \mathscr{P}_{q q, R}^{(\varepsilon)}(z)+\mathscr{O}\left(\varepsilon^{2}\right)$ with $\hat{P}_{q q}^{(0)}(z)$ the leading-order (LO) AltarelliParisi splitting kernel, and the expression for $\mathscr{P}_{q q, R}^{(\varepsilon)}(z)$ is given in Ref. [1]. I have also introduced

$$
\left[\alpha_{s}\right]=\frac{\alpha_{s}(\mu)}{2 \pi} \frac{\mu^{2 \varepsilon} e^{\varepsilon \gamma_{E}}}{\Gamma(1-\varepsilon)} .
$$

A similar approach may be followed for the collinear limit $C_{42}$. The pole structure is remarkably straightforward; indeed, the poles proportional to $F_{L M}(1,2)$ cancel against the poles from the virtual amplitude, while the poles proportional to convolutions of $F_{L M}$ with the splitting function cancel against the pdf renormalization.

After cancelling the poles, one can take the limit $\varepsilon \rightarrow 0$ and compute all quantities in four dimensions. Including the virtual corrections and the pdf renormalization terms, the renormalized differential cross section at NLO is

$$
\begin{aligned}
2 s \cdot \mathrm{d} \hat{\sigma}^{\mathrm{NLO}}= & \left\langle F_{L V}^{\mathrm{fin}}(1,2)+\frac{\alpha_{s}(\mu)}{2 \pi}\left[\frac{2}{3} \pi^{2} C_{F} F_{L M}(1,2)\right]\right\rangle+\left\langle\hat{O}_{\mathrm{NLO}} F_{L M}(1,2,4)\right\rangle \\
& +\frac{\alpha_{s}(\mu)}{2 \pi} \int_{0}^{1} \mathrm{~d} z\left[\ln \frac{s}{\mu^{2}} \hat{P}_{q q}^{(0)}(z)-\mathscr{P}_{q q, R}^{(\varepsilon)}(z)\right]\left\langle\frac{F_{L M}(z \cdot 1,2)}{z}+\frac{F_{L M}(1, z \cdot 2)}{z}\right\rangle,
\end{aligned}
$$


where $\hat{O}_{\mathrm{NLO}}=\left(I-S_{4}\right)\left(I-C_{41}-C_{42}\right)$. Equation (2.9) is the sum of lower multiplicity terms, with or without convolutions with splitting functions; a real emission term, whose singular configurations have been removed by nested subtractions; and a finite remainder of the virtual amplitude. The aim now is to devise a similar procedure and obtain an analogous result at NNLO.

\section{Next-to-next-to-leading order calculation}

Having studied nested soft-collinear subtractions at NLO, we are now in a position to consider the NNLO calculation. I am interested in the double-real emission contribution. ${ }^{1}$ The differential cross section from $q_{1} \bar{q}_{2} \rightarrow V+g_{4} g_{5}$ is

$$
2 s \cdot \mathrm{d} \sigma^{\mathrm{RR}}=\frac{1}{2 !} \int\left[\mathrm{d} g_{4}\right]\left[\mathrm{d} g_{5}\right] F_{L M}(1,2,4,5) .
$$

The singularity structure of this contribution is much more complicated than at NLO: either $g_{4}$ or $g_{5}$ may become soft, or either may become collinear to either initial state parton, or $g_{4}$ and $g_{5}$ may become collinear to each other. A combination of these configurations may also occur, so one needs to consider different approaches to each limit. The key to extracting the poles is to separate these singular regions.

In doing so, I will make use of an important feature of onshell, gauge invariant QCD scattering amplitudes, namely color coherence. A simple explanation of this property is as follows. Consider the emission of a soft parton from a hard parton which subsequently undergoes multiple splittings, which may or may not be collinear. The wavelength of the soft parton is too large to resolve the details of the splitting; it only "sees" the total color charge of all the partons resulting from the splitting. Thus soft and collinear configurations decouple and can be treated independently of one another. In the context of a subtraction scheme, this means that soft and collinear limits can be removed separately. Therefore, energies and angles may be independently parametrized, without the need for energy-angle ordering. Not only does this provide greater flexibility in the numerical implementation of the subtraction scheme, but also allows us to reduce the number of required sectors from five to four.

I will now describe the treatment of the double-real emission singularities in the nested softcollinear subtraction scheme. I begin by introducing additional operators for the double-soft and double-collinear limits

$$
\begin{aligned}
\mathbb{S} A & =\lim _{E_{4}, E_{5} \rightarrow 0} A, \text { at fixed } E_{5} / E_{4} \\
\mathbb{C}_{i} A & =\lim _{\rho_{4 i}, \rho_{5 i} \rightarrow 0} A, \text { with non vanishing } \rho_{4 i} / \rho_{5 i}, \rho_{45} / \rho_{4 i}, \rho_{45} / \rho_{5 i} .
\end{aligned}
$$

I then order the gluon energies by imposing $E_{4}>E_{5}$, and define

$$
2 s \cdot \mathrm{d} \sigma^{\mathrm{RR}}=\int\left[d g_{4}\right]\left[d g_{5}\right] \theta\left(E_{4}-E_{5}\right) F_{L M}(1,2,4,5) \equiv\left\langle F_{L M}(1,2,4,5)\right\rangle,
$$

where the ordering of the gluon energies has removed the $1 / 2$ ! symmetry factor. As at NLO, the gluon energies $E_{4}$ and $E_{5}$ are bounded from above by $E_{\max }$, and the bounds on the gluon energies are imposed in the partonic center-of-mass frame.

\footnotetext{
${ }^{1}$ The details of other contributions, e.g. the double-virtual or real-virtual, are discussed in depth in Ref. [1], and are not considered in these proceedings.
} 
As a result of the energy ordering $E_{4}>E_{5}$, soft singularities only occur when either $g_{5}$ is soft or when both $g_{4}$ and $g_{5}$ are soft. The soft singularities may then be removed by writing

$$
\begin{aligned}
\left\langle F_{L M}(1,2,4,5)\right\rangle & =\left\langle\mathbb{S} F_{L M}(1,2,4,5)\right\rangle+\left\langle S_{5}(I-\mathbb{S}) F_{L M}(1,2,4,5)\right\rangle \\
& +\left\langle\left(I-S_{5}\right)(I-\mathbb{S}) F_{L M}(1,2,4,5)\right\rangle .
\end{aligned}
$$

The first term on the left-hand side in Eq. (3.4) corresponds to the double-soft limit, in which both gluons decouple completely. The second term captures the limit where $g_{5}$ is soft but singularities from $S_{4}$ are removed. The final term has all soft singularities removed. All three terms, however, contain (potentially overlapping) collinear singularities, which must be disentangled.

I will tackle the collinear singularities in two steps. First, I partition the phase space by writing

$$
w^{14,15}+w^{24,25}+w^{14,25}+w^{15,24}=1 .
$$

The expressions for the partitions $w$ in terms of the angles between the partons are given in Ref. [1] and are not repeated here. The important point is that these factors vanish in various collinear limits, and as a result only certain limits need to be considered in each partition. The triple-collinear partitions $w^{i 4, i 5}$ (for $i=1,2$ ) only have singularities corresponding to operators $C_{4 i}, C_{5 i}$ and $C_{45}$, while the double-collinear partitions $w^{14,25}$ and $w^{15,24}$ only have singularities corresponding to, respectively, $C_{41}$ and $C_{52}$, and $C_{51}$ and $C_{42}$.

The double-collinear partitions are now free of overlapping singularities, but this not yet the case for the triple-collinear partitions. I therefore define an angular ordering, e.g. in partition $w^{14,15}$

$$
\begin{aligned}
1 & =\theta\left(\eta_{51}<\frac{\eta_{41}}{2}\right)+\theta\left(\frac{\eta_{41}}{2}<\eta_{51}<\eta_{41}\right)+\theta\left(\eta_{41}<\frac{\eta_{51}}{2}\right)+\theta\left(\frac{\eta_{51}}{2}<\eta_{41}<\eta_{51}\right) \\
& \equiv \theta^{(a)}+\theta^{(b)}+\theta^{(c)}+\theta^{(d)} .
\end{aligned}
$$

which divides the triple-collinear partitions into four sectors, each of which only contains one collinear limit. Sectors $a$ and $c$ have limits $C_{51}$ and $C_{41}$, respectively, while sectors $b$ and $d$ only have the $C_{45}$ limit. Note that sectors $b$ and $d$ are not the same, as the energy of the gluons are ordered. The partition $w^{24,25}$ may be treated in a similar manner.

I have now split up the phase space in such a way that there are no overlapping singularities. The collinear singularities can therefore be subtracted in a straightforward way in each partition and sector, in a similar manner to what was done at NLO. The third term of Eq. (3.4) can be written as

$$
\left\langle\left(I-S_{5}\right)(I-\mathbb{S}) F_{L M}(1,2,4,5)\right\rangle=\left\langle F_{L M}^{S_{r} c_{s}}(1,2,4,5)\right\rangle+\left\langle F_{L M}^{S_{r} c_{t}}(1,2,4,5)\right\rangle+\left\langle F_{L M}^{s_{r} c_{r}}(1,2,4,5)\right\rangle .
$$

The full expressions for the three terms on the right-hand side of the above equation are given in Ref. [1] and are not repeated here. It suffices to note that the third term has all singularities - soft and collinear - removed by nested subtractions. As a result, it can be evaluated in four dimensions and integrated numerically. It is the only term which involves the fully-resolved double-real matrix element. The first two terms of Eq. (3.7) contain the single-collinear and triple-collinear singularities, respectively. Together with the first two terms of Eq. (3.4) these are the integrated subtraction terms, which completely describe the singular structure of the double-real emission. In each of these four terms, the unresolved gluons decouple either partially (in the case of a collinear 
singularity) or completely (in the case of a soft singularity). If the gluon decouples completely, one can integrate over its energy and angles. If the gluon decouples partially, one can integrate over its angles and rewrite its energy integral as a convolution with a splitting function. The resulting expressions contain explicit poles in $1 / \varepsilon$ multiplying matrix elements of lower multiplicity convoluted with splitting functions, which can then be cancelled against poles coming from the other NNLO corrections (e.g. the double-virtual).

The above procedure has the drawback that one is left with expressions from multiple partitions and sectors. However, it turns out that combining sectors in a judicious manner can lead to dramatic simplifications. I will sketch one such simplification here, which appears in the doublecollinear partition

$$
\begin{aligned}
D C= & \left\langle[I-\mathbb{S}]\left[I-S_{5}\right]\left[\left(C_{41}\left[\mathrm{~d} g_{4}\right]+C_{52}\left[\mathrm{~d} g_{5}\right]\right) w^{14,25}+\left(C_{42}\left[\mathrm{~d} g_{4}\right]+C_{51}\left[\mathrm{~d} g_{5}\right]\right) w^{15,24}\right]\right. \\
& \left.\times F_{L M}(1,2,4,5)\right\rangle .
\end{aligned}
$$

Note that the collinear limits are defined to act on the phase space measures which are explicitly written to their right. The first term involves the limit $C_{41}$, so $g_{4}$ decouples partially. Upon integrating over the phase space of $g_{4}$ I obtain

$$
\begin{aligned}
\left\langle[I-\mathbb{S}]\left[I-S_{5}\right] C_{41}\left[\mathrm{~d} g_{4}\right] w^{14,25} F_{L M}(1,2,4,5)\right\rangle= & -\frac{\left[\alpha_{s}\right] s^{-\varepsilon}}{\varepsilon} \int_{z_{\min }}^{z_{\max }\left(E_{5}\right)} \frac{\mathrm{d} z}{(1-z)^{1+2 \varepsilon}} \mathscr{P}_{q q}(z) \\
& \times\left\langle\tilde{w}_{4|| 1}^{14,25}\left[I-S_{5}\right] F_{L M}(z \cdot 1,2,5)\right\rangle,
\end{aligned}
$$

with $\tilde{w}_{4 \| 1}^{14,25}=\lim _{\rho_{14} \rightarrow 0} w^{14,25}$. The limits of integration $z_{\min }=1-E_{\max } / E_{1}$ and $z_{\max }\left(E_{5}\right)=1-$ $E_{5} / E_{1}$ follow from the definition $z=1-E_{4} / E_{1}$ and the restrictions $E_{\max }>E_{4}>E_{5}$. Eq. (3.9) should be written as a convolution with a splitting function, so ideally the integration should run over $0<z<1$. The lower integration limit can be extended to 0 , since if $z<z_{\min }$ there is not sufficient energy to produce the color singlet final state, but the upper limit cannot be extended in this manner. However, if I now look at the fourth term, I find

$$
\begin{aligned}
\left\langle[I-S S]\left[I-S_{5}\right] C_{51}\left[\mathrm{~d} g_{5}\right] w^{15,24} F_{L M}(1,2,4,5)\right\rangle= & -\frac{\left[\alpha_{s}\right] s^{-\varepsilon}}{\varepsilon} \int_{z_{\min }\left(E_{4}\right)}^{1} \frac{\mathrm{d} z}{(1-z)^{1+2 \varepsilon}} \hat{\mathscr{P}}_{q q}^{(-)}(z) \\
& \times\left\langle\tilde{w}_{5 \| 1}^{15,24} F_{L M}(z \cdot 1,2,4)\right\rangle,
\end{aligned}
$$

where I have now defined $z=1-E_{5} / E_{1}$ leading to the requirement $z>z_{\min }\left(E_{4}\right)=1-E_{4} / E_{1}$. I now rename the resolved gluon in Eq. (3.9) $g_{5} \rightarrow g_{4}$ and combine this with Eq. (3.10), finding

$$
\begin{aligned}
\left\langle[I-\mathbb{S}]\left[I-S_{5}\right]\left[\left(C_{41}\left[\mathrm{~d} g_{4}\right] w^{14,25}+C_{51}\left[\mathrm{~d} g_{5}\right] w^{15,24}\right) F_{L M}(1,2,4,5)\right\rangle\right. & \\
= & -\frac{\left[\alpha_{s}\right] s^{-\varepsilon}}{\varepsilon} \int_{0}^{1} \frac{\mathrm{d} z}{(1-z)^{1+2 \varepsilon}}\left\langle\tilde { w } _ { 5 \| 1 } ^ { 1 5 , 2 4 } \left(\hat{\mathscr{P}}_{q q}^{(-)}(z)\left[I-S_{4}\right] F_{L M}(z \cdot 1,2,4)+\right.\right. \\
& \left.\left.\theta\left(z_{4}-z\right) 2 C_{F}\left[I-S_{4}\right] F_{L M}(1,2,4)+\theta\left(z_{4}-z\right) \hat{\mathscr{P}}_{q q}^{(-)}(z) S_{4} F_{L M}(z \cdot 1,2,4)\right)\right\rangle,
\end{aligned}
$$




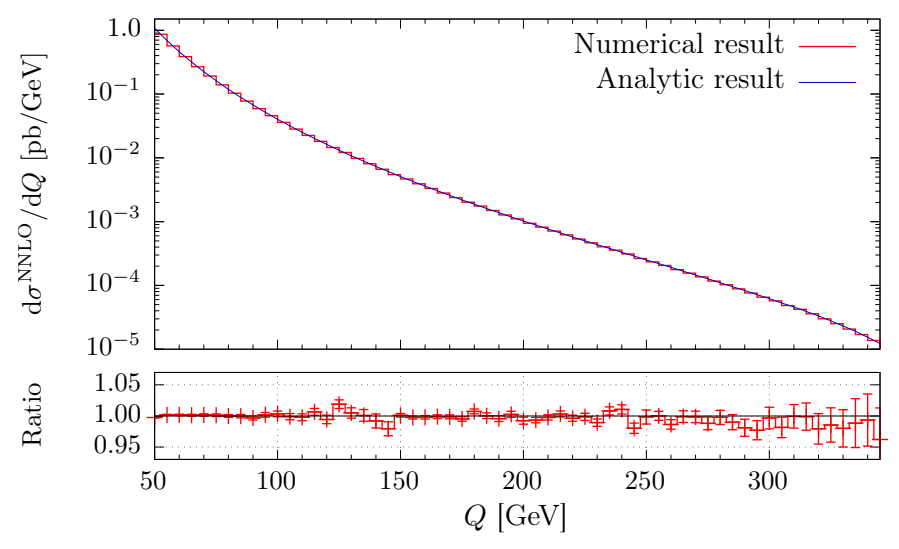

Figure 1: Comparison of the NNLO QCD contribution $\mathrm{d} \sigma^{\mathrm{NNLO}} / \mathrm{d} Q$ computed using the nested softcollinear subtraction scheme with the analytic results in Ref. [15].

where the integration runs from 0 to 1 , as desired. The situation is still not ideal since the splitting functions in Eqs. (3.9) and (3.10) are different, and this results in the restrictions on $z$ from the $\theta$-functions in Eq. (3.11). However, if one combines this result from the double-collinear partition with results from the triple-collinear partition, one sees further simplifications which remove the need for any restrictions. I refer interested readers to Ref. [1] for more details.

Before displaying the results of a proof-of-principle calculation, it is useful to recapitulate this section. I considered the double-real corrections to color singlet production $q \bar{q} \rightarrow V+g g$, and regulated the soft singularities first and then the collinear singularities. It is possible to treat soft and collinear singularities independently because of the color coherence property of gauge invariant QCD amplitudes. The phase space is then decomposed into sectors to ensure that there are no overlapping collinear singularities. The singularities in each sector may be removed through nested subtractions, leading to a finite piece which can be evaluated in four dimensions, as well as integrated subtraction terms which contain lower-multiplicity matrix elements convoluted with splitting functions. The $1 / \varepsilon$ poles are made explicit in the integrated subtration terms, and these can be cancelled against the poles from other NNLO contributions. Indeed, this cancellation occurs within each different particle multiplicity. I note that, since the double-soft and triple-collinear singularities are evaluated numerically, the cancellation is also performed numerically. The situation at NNLO is thus identical to that at NLO, as was the goal.

\section{Numerical results}

In this section I present NNLO QCD results for the process $p p \rightarrow \gamma^{*}+X \rightarrow e^{-} e^{+}+X$ at the 14 $\mathrm{TeV}$ LHC. This process is particularly well-suited to a proof-of-concept calculation since analytic results for each partonic channel at NNLO have been known for some time [15]. I again focus on the channel $q \bar{q} \rightarrow \gamma^{*}+n g$, but all other partonic channels display a similar level of agreement to what we show below. I include lepton pairs with an invariant mass $50 \mathrm{GeV}<Q<350 \mathrm{GeV}$. I use NNPDF3.0 parton distribution functions [16] and take the renormalization and factorization scale $\mu=100 \mathrm{GeV}$. 
The NNLO contributions for this channel, from our calculation and from the analytic results of Ref. [15], are

$$
\mathrm{d} \sigma^{\mathrm{NNLO}}=14.471(4) \mathrm{pb} \quad \mathrm{d} \sigma_{\text {analytic }}^{\mathrm{NNLO}}=14.470 \mathrm{pb},
$$

in agreement below the level of one per-mille. I display the differential cross section in $Q$ in Fig. 1, in which we see that the level of agreement is at the level of a few per-mille to a few percent across five orders of magnitude. This level of agreement on the NNLO contribution translates into almost absolute precision for the physical cross sections and kinematic distributions.
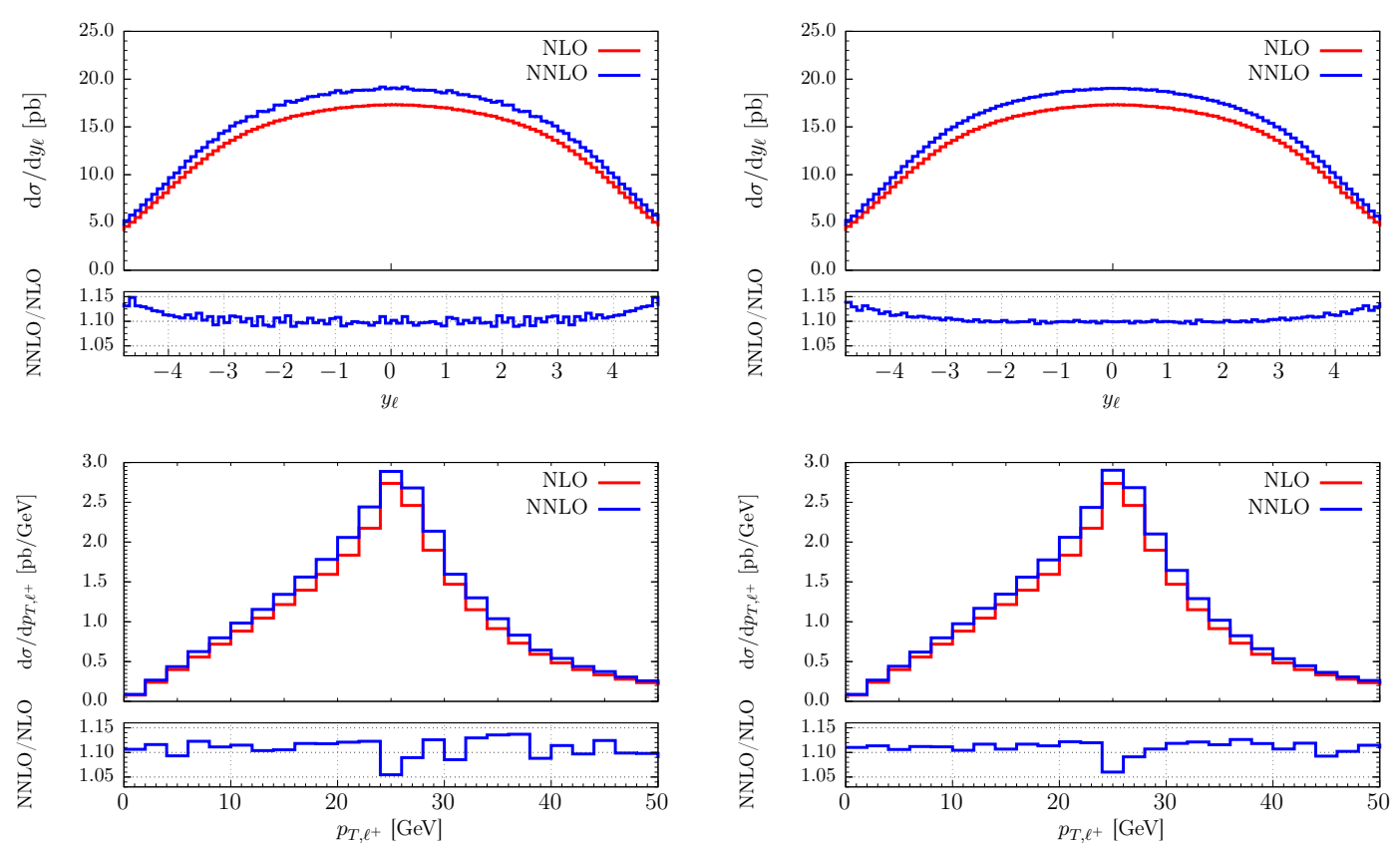

Figure 2: Rapidity distribution of the lepton (upper row) and $p_{T}$ distribution of the lepton (lower row) at different orders of perturbation theory. The upper panes show the differential distributions, the lower panes shown the NNLO/NLO ratio for a given observable. Plots on the left show results from a runtime of $\mathscr{O}(10)$ CPU hours; plots on the right show results from a runtime of $\mathscr{O}(100)$ CPU hours.

As mentioned in the introduction, one of the important issues for NNLO calculations is their practicality, and in particular their ability to produce high precision predictions in the presence of fiducial cuts imposed by experiments. To illustrate this aspect of our subtraction scheme, I show the differential distributions of the rapidity and transverse momentum of the lepton in Fig. 2. The figures on the left are obtained with $\mathscr{O}(10)$ hours of CPU runtime; those on the right with $\mathscr{O}(100)$ hours of CPU runtime. My purpose here is not to investigate the exact CPU requirements of the subtraction scheme but rather to get an overall idea of the time taken to produce results with different precision requirements. The lepton rapidity distribution has bin-to-bin fluctuations at the percent level after only $\mathscr{O}(10)$ CPU hours of runtime, and increasing this to $\mathscr{O}(100)$ hours of CPU runtime decreases the bin-to-bin fluctuations to the permille level. The transverse momentum of the lepton is less stable, and $\mathscr{O}(100)$ hours of CPU runtime are required to obtain percent-level stability. I point out that the feature at $p_{T, \ell^{+}}=25 \mathrm{GeV}$ is physical and not a statistical fluctuation, caused by the smearing of the peak due to the NNLO corrections. A similar feature is found when 
comparing LO and NLO results. The less stable behavior of the transverse momentum distribution reflects that this is quite a delicate observable, a result of the fact that it receives contributions from a wide range of values of invariant mass $Q$. The stability of this observable is expected to improve once the Z-boson propagator is introduced, as this should localize the bulk of the cross sections in a smaller range of $Q$ values. However, I note that even without this, the subtraction scheme allows for high precision computations while also delivering phenomenologically acceptable results from very short runtimes.

\section{Conclusion}

In these proceedings, I have presented a new NNLO subtraction scheme, based on the sectordecomposition framework for extending FKS subtraction to NNLO. The scheme is characterized by the independent treatment of the soft and collinear limits. We have developed a nested subtraction procedure, resulting in a manifestly regulated finite term and integrated subtraction terms which are expressed as convolutions of splitting functions with lower multiplicity matrix elements. The integrated subtraction terms have explicit poles in $1 / \varepsilon$ allowing the singularities to be cancelled within each lower-multiplicity structure. Although I have presented results for one particular partonic channel in these proceedings, we have tested the subtraction scheme in Drell-Yan and $W$-boson production for all partonic channels, as well as in the decay $H \rightarrow b \bar{b}$, and found excellent agreement with previous results. The details of these calculations will be presented in a forthcoming publication.

Acknowledgments: I thank the organizers of Radcor 2017 for a well-organized and stimulating conference in a truly beautiful setting. I am grateful for support from the German Federal Ministry for Education and Research (BMBF) under grant 05H15VKCCA.

\section{References}

[1] F. Caola, K. Melnikov and R. Röntsch, Eur. Phys. J. C 77 (2017) no.4, 248

[2] S. Catani and M. Grazzini, Phys. Rev. Lett. 98, 222002 (2007).

[3] M. Grazzini, JHEP 0802 (2008) 043.

[4] R. Boughezal, C. Focke, X. Liu and F. Petriello, Phys. Rev. Lett. 115 (2015) no.6, 062002.

[5] J. Gaunt, M. Stahlhofen, F. J. Tackmann, and J. R.Walsh, JHEP 09 (2015), 058.

[6] A. Gehrmann-De Ridder, T. Gehrmann and E. W. N. Glover, JHEP 0509 (2005), 056; Phys. Lett. B 612 (2005), 49; Phys. Lett. B 612 (2005) 36; A. Daleo, T. Gehrmann and D. Maitre, JHEP 0704 (2007), 016; A. Daleo, A. Gehrmann-De Ridder, T. Gehrmann and G. Luisoni, JHEP 1001 (2010), 118; T. Gehrmann and P.F. Monni, JHEP 1112 (2011), 049; R. Boughezal, A. Gehrmann-De Ridder and M. Ritzmann, JHEP 1102 (2011), 098; A. Gehrmann-De Ridder, T. Gehrmann and M. Ritzmann, JHEP 1210 (2012) 047; J. Currie, E.W.N. Glover and S. Wells, JHEP 1304 (2013) 066.

[7] M. Czakon, Phys. Lett. B 693 (2010) 259; M. Czakon, Nucl. Phys. B 849 (2011) 250.

[8] M. Czakon and D. Heymes, Nucl. Phys. B 890 (2014) 152.

[9] R. Boughezal, K. Melnikov and F. Petriello, Phys. Rev. D 85 (2012) 034025. 
[10] M. Cacciari, F. A. Dreyer, A. Karlberg, G. P. Salam and G. Zanderighi, Phys. Rev. Lett. 115 (2015) no.8, 082002 .

[11] V. Del Duca, C. Duhr, A. Kardos, G. Somogyi and Z. Trocsanyi, Phys. Rev. Lett. 117 (2016) no.15, 152004; V. Del Duca, C. Duhr, A. Kardos, G. Somogyi, Z. Szor, Z. Trocsanyi and Z. Tulipant, Phys. Rev. D 94 (2016) no.7, 074019.

[12] S. Frixione, Z. Kunszt and A. Signer, Nucl. Phys. B 467 (1996) 399.

[13] S. Frixione, Nucl. Phys. B 507 (1997) 295.

[14] S. Catani and M. H. Seymour, Nucl. Phys. B 485 (1997) 291 Erratum: [Nucl. Phys. B 510 (1998) 503]

[15] R. Hamberg, W. L. van Neerven and T. Matsuura, Nucl. Phys. B 359 (1991) 343 Erratum: [Nucl. Phys. B 644 (2002) 403].

[16] R. D. Ball et al. [NNPDF Collaboration], JHEP 1504 (2015) 040.

[17] S. Alioli et al., [arXiv:1606.02330 [hep-ph]]. 